ISSN: $1130-3743$

\title{
LA COMPLEJIDAD DE LAS RELACIONES TEORÍA-PRÁCTICA EN EDUCACIÓN
}

\author{
The Complex Relationship Between Theory and Practice in \\ Education
}

La complexité des relations théorie-pratique en éducation

María ClemENTE LINUESA

Universidad de Salamanca. Facultad de Educación. Departamento de Didáctica, Organización Escolar y Métodos de Investigación. Paseo de Canalejas, 169.

37008 Salamanca.Correo-e: mcl@usal.es

Fecha de recepción: enero de 2007

Fecha de aceptación definitiva: abril de 2007

BIBLID [(1130-3743) 19, 2007, 25-46]

RESUMEN

En este trabajo se pretende hacer un análisis sobre cuáles son las relaciones entre las teorías y las prácticas educativas, tomando como ejemplo el caso de la enseñanza del lenguaje escrito. En primer lugar se plantea un somero repaso por los conceptos de teoría y de práctica en educación, analizando cuáles son sus dependencias y cómo puede entenderse esta relación dado el carácter intencional que caracteriza toda acción educativa. Centrándose en el caso del lenguaje escrito, se alude a los ámbitos en que se genera el conocimiento pedagógico y los distintos agentes que ejercen influencia en su configuración: la investigación, la propia práctica educativa, los valores y prácticas sociales e incluso, muchas veces, las políticas educativas. Se concluye con una breve síntesis sobre qué componentes sustentan una teoría sobre la enseñanza de la lengua escrita.

Palabras clave: teoría, práctica, conocimiento pedagógico. 
SUMMARY

The aim of this work is to provide an analysis of the relationships between education theory and practice, taking the case of written language as an example. First, we offer a brief summary of the concepts of educational theory and practice, analysing their dependencies and how this relationship might be understood in light of the intentional nature that characterises all educational actions. Focusing on the case of written language, we refer to the environments in which pedagogical knowledge is generated and the different agents affecting its configuration; research, educational practices themselves, social values and practices and, often, educational policies. We conclude with a brief synthesis of which components uphold a theory of written language teaching.

Key words: theory, practice, pedagogical kowledge.

\section{SOMMAIRE}

Dans ce travail, une analyse sur les relations existantes entre les théories et les pratiques éducatives est présentée à partir de l'exemple de l'enseignement de la langue écrite. En premier lieu, une brève révision des concepts de théorie et de pratique en éducation est réalisée en analysant leurs dépendances et la manière dont cette relation peut être comprise étant donné le caractère intentionnel qui caractérise toute action éducative. À partir du cas du langage écrit, on observe les domaines dans lesquels la connaissance pédagogique naît et les différents agents ayant une influence sur sa configuration: la recherche, la pratique éducative elle-même, les valeurs et les pratiques sociales et -très souvent- les politiques éducatives. Finalement, une brève synthèse sur les composantes qui se trouvent à la base d'une théorie sur l'enseignement de la langue écrite est proposée.

Mots clés: théorie, pratique, connaissance pédagogique.

\section{INTRODUCCIÓN}

En educación han existido visiones diversas y cambios importantes respecto del valor que la teoría ha tenido para la práctica y en general sobre la relación teoría-práctica. Cuestiones como: qué carácter debe tener una teoría educativa, quién genera conocimiento teórico en educación, qué conocimiento es "útil" para la práctica, etc., están en el origen de muchas cuestiones que han preocupado y siguen preocupando tanto al gremio de los docentes como, sobre todo, al de los teóricos.

Desde posiciones cientifistas, que desvinculaban claramente la teoría de la práctica, dando a la primera un valor primordial para fundamentar a la segunda, se ha pasado a partir de los ochenta a defender por algunos que la única teoría válida es la que se genera en la práctica Carr (1996). La primera posición asumía y defendía que el conocimiento teórico era generado por expertos y provenía incluso 
de materias diferentes a la propia educación, como la psicología, la sociología o la filosofía. La segunda sostiene que el único conocimiento pedagógico valioso es el que se genera en la acción mediante procesos de reflexión sobre la práctica.

Vinculada a esas cuestiones, otra pregunta relevante radica en conocer qué tipo de conocimiento rige las prácticas de los profesores, lo que denominamos conocimiento práctico del profesor, cómo llegan a configurarlo y qué vinculación tiene con las teorías de la enseñanza.

En todo caso, las teorías de la enseñanza tienen particularidades respecto de otro tipo de conocimiento teórico dado su carácter axiológico y práctico, es un tipo de conocimiento que supone un saber qué vinculado a un saber cómo y a un saber para qué.

En este trabajo nos hemos planteado algunas reflexiones sobre el carácter de las teorías de la enseñanza, así como sobre las fuentes desde las que se generan, tomando como punto de referencia el ejemplo de la enseñanza de la lengua escrita, pues creemos que es un caso interesante para mostrar las particularidades y complejidades de las teorías educativas en general.

Conviene dejar claro que cuando hablamos de enseñanza del lenguaje escrito hacemos referencia al conjunto de estrategias que llevan al aprendiz a dominar la lengua escrita. Ello implica tener claro para qué leemos, funciones del lenguaje escrito, y dominar el sistema de escritura correspondiente. En el caso de los alfabetos, tal sistema es básicamente una representación de la lengua oral, que tiene una estructura y unos componentes determinados, también de orden ortográfico.

Así pues, nos proponemos analizar, por una parte, qué componentes sustentan una teoría sobre la enseñanza de la lengua escrita. Por otra, analizaremos los ámbitos en que se genera el conocimiento pedagógico y los distintos agentes que ejercen influencia en su configuración: la investigación, la propia práctica educativa, los valores y prácticas sociales e incluso, muchas veces, las políticas educativas. Antes realizaremos un somero repaso por los conceptos de teoría y de práctica en educación. Entendiendo por teoría el conjunto organizado de conocimientos de una disciplina.

\section{TEORÍA Y PRÁCTICA: RELACIONES Y DEPENDENCIAS}

Los conceptos de teoría y práctica ostentan en educación distintos sentidos y significados dependiendo de los contextos en que se usen. Por ejemplo, podemos aludir a la cuestión sobre la dimensión explicativa de las teorías, también a la relación entre conocer y actuar. Respecto a este último término nos planteamos no sólo el cómo, sino el porqué y el para qué de la actuación. Esta relación parece compleja, y no sólo lo es en el ámbito educativo, de hecho es una problemática planteada constantemente en los círculos epistemológicos (Hacking, 1996).

El término teoría ha tenido y tiene significados diversos. Ha sido entendida como conocimiento desinteresado, saber por saber, sin sentido práctico ni aplicado, 
así se interpreta desde posiciones idealistas. Desde la perspectiva marxista, antítesis del idealismo, la búsqueda del conocimiento, e incluso de la verdad, sólo tiene lugar en la praxis, siendo, por tanto, las relaciones entre teoría y práctica ineludibles. Otra gran visión es la del positivismo de los siglos diecinueve y veinte, que sólo ha visto en la teoría el valor de sus aplicaciones, de sus resultados prácticos.

El uso del término práctica tampoco es unívoco en las distintas tradiciones filosóficas. En todo caso en educación podemos entenderlo como una praxis que implica conocimiento para conseguir determinados fines. La práctica es el saber hacer, tanto si lo realizamos materialmente como si no.

La posición idealista de la teoría de la educación, que vincula a ésta con lo que se denomina ciencias culturales frente a ciencias naturales, supone que la educación se caracterizara por investigar unas realidades concretas no generalizables a través de métodos especulativos, alejándose del método científico-experimental. La cuestión es que al vincularse a filosofías idealistas dio lugar, como señalan Colom y Rincón (2004) a que se ontologizara la educación alejándola de su realidad inmediata. Tal hecho no tiene que ver, según estos autores, con la identidad epistemológica de la pedagogía, sino con una cuestión genealógica, con su vinculación histórica a esas posiciones idealistas.

Como antítesis a esta posición, el punto de vista aplicado se ha basado en la idea, según la cual, la teoría constituye un conjunto de leyes, enunciados e hipótesis que configuran un corpus de conocimiento científico, sistematizado y organizado, que permite derivar a partir de estos fundamentos reglas de actuación. Como explican Colom y Rincón (2004), supone admitir una relación de supremacía de la teoría respecto de la práctica.

El afán experimentalista aspiraba a desarrollar un cuerpo unívoco de conocimientos sobre el hecho educativo... con la pretensión de describir y explicar la fenomenología educativa (leyes generales) y deducir de dicho conocimiento científico los procedimientos y técnicas que pudieran regular de manera eficaz las prácticas de enseñanza (normas particulares) (Colom y Rincón, 2004, 38).

Esta visión, sostenida durante largo tiempo, y de carácter pretendidamente universalista, está tomada de las ciencias naturales y ha sido aplicada a la educación obedeciendo al planteamiento según el cual se necesita un conocimiento científico que guíe y legitime la intervención educativa, la práctica, entendida, por ello, como una aplicación de dicha teoría. Tal planteamiento se desarrolla en los ochenta y tiende a identificar la teoría de la educación como un saber o conocimiento aplicable cuyo objetivo es la mejora.

Esta visión supone asumir que la teoría tiene un componente normativo desde una justificación científico-tecnológica, vinculándose así el concepto de teoría al de tecnología. Sarramona (2003) señala tal punto de vista del modo siguiente: «El modo de proceder de la teoría de la educación para resolver los problemas educativos de la práctica debería ser de naturaleza tecnológica" (Sarramona, 2003, 168). Este autor realiza una compilación de opiniones bastante coincidentes que 
relacionan el concepto de teoría de la educación, entendida como ciencia aplicada, con el de tecnología. El común denominador de dicha visión es considerar el acto pedagógico como tecnológico, en el que se vinculan los elementos teóricos, axiológicos y la acción educativa. De hecho, hace alusión a la obra de T. Moore Educational Theory: An introduction en la que se resalta que lo importante de la teoría de la educación es que pueda servir para la práctica.

Esta última perspectiva tiene, para otros autores, sobre todo los vinculados a la teoría crítica, serios inconvenientes respecto de la relación teoría-práctica educativa, como, por otra parte, ocurre en otras ciencias sociales (Carr, 1996). Por un lado, su pretendido carácter generalizador choca con las variadas realidades educativas, con las diferentes situaciones, en las que aplicar un conocimiento que se presupone universalmente válido. La diversidad de situaciones escolares y educativas en general es tanta y está condicionada por factores tan complejos que difícilmente una sola teoría podría servir de base para una intervención realista. Además se ha ampliado notablemente lo que tradicionalmente se ha considerado lo educativo. Esto es reconocido en todo caso se comparta o no una posición crítica. Autores como Carrasco y García del Dujo (2002) resaltan la idea de que ha existido un reduccionismo según el cual el proceso educativo se asimilaba únicamente a los procesos de enseñanza-aprendizaje en contextos institucionales, algo que actualmente no se admitiría.

Por otra parte, aunque los motivos en ciencias sociales, y por ello en la educación, pudieran ser más evidentes, este ideal racionalista de ciencia general se contempla cada vez más como una utopía. Como expresa Cartwright (1999), el conocimiento científico se parece más a una realidad moteada que a una estructura piramidal. Es decir, que la ciencia tiene que ver más con estudios sobre sectores diferenciados de la realidad que con la idea de una ciencia unificada.

Las posiciones mayoritariamente asumidas hoy sostienen que la relación entre teoría y práctica no podemos entenderla como una dependencia de la práctica respecto de la teoría. Tampoco podemos reducir el concepto de teoría al de conocimiento científico, ni a un solo tipo de conocimiento, ni a una única teoría. Por un lado, en la propia acción se pueden generar procesos de conocimiento, asunto este muy complejo, en el que no entraremos apenas en este trabajo. Por otro lado, como queremos mostrar, el conocimiento teórico, que usan los prácticos, procede de ámbitos diversos. Además las teorías en educación tienen un componente intencional y axiológico que determina en gran medida las prácticas docentes.

En el caso del conocimiento teórico sobre la enseñanza del lenguaje escrito, aun tratándose de un asunto relativamente concreto, encontraremos ejemplos de lo expuesto. Contamos con teorías sobre el aprendizaje de la lectura, que pueden ser valiosas, pero son parciales. Se han ocupado de ofrecer conocimiento sobre el hecho lector pero de forma incompleta porque las cuestiones, las preguntas que se han formulado no han abarcado los diferentes aspectos que intervienen en el aprendizaje de la lengua escrita. Así el conocimiento teórico que podemos agrupar en la perspectiva comunicativa se ha planteado fundamentalmente la cuestión 
funcional de la lectura, cómo hacer captar al que aprende lo interesante y necesario que es leer y escribir, qué usos tendrá la escritura para un sujeto determinado en una determinada comunidad social y cultural. Lo que se propugna a través de estas teorías es introducir al sujeto en el mundo de la lectura, hacerle ver su importancia, sumergirle en un contexto letrado. Muy al contrario, la teoría psicolingüística se ha ocupado profusamente y de forma casi exclusiva de explicar los problemas que tienen muchos sujetos para apropiarse del código, y por ello del modo como ayudarles a lograrlo, ha planteado modelos bien respaldados por datos sobre los procesos que intervienen en la lectura como actividad cognitiva, pero ha obviado preguntarse por qué un sujeto querría, incluso debería aprender a leer, en definitiva, se han despreciado todos los aspectos socioculturales que implica apropiarse de esa herramienta cultural. En unos casos interesaba responder a la pregunta: qué es leer desde el punto de vista cognitivo y en otros: para qué leer.

Esto nos lleva a plantear precisamente el papel de las intenciones, y no sólo los conocimientos en la configuración de las decisiones prácticas en la educación, dado que en la acción educativa, por ser una acción humana, las intenciones, las metas, son un elemento constitutivo de esa acción, tan importante como el conocimiento mismo. Lo que valoramos como objeto de educación tiene que ver tanto o más con deseos y nuevas metas que con el pasado y lo sabido. Conocer las intenciones educativas puede encaminar a tomar decisiones en la práctica que no vendrán determinadas exclusivamente por el saber teórico acumulado, por el conocimiento científico, aunque exista y sea parte esencial, entre otros elementos, de los fundamentos de tales prácticas.

Siguiendo con el ejemplo, las intenciones, los objetivos, los valores, el sentido del lector en cada situación pueden hacer variar las prácticas que llevemos a cabo. Si contáramos sólo con el conocimiento teórico derivado de la investigación psicolingüística de corte cognitivo que, sin duda, ha esclarecido muchos asuntos relativos a los procesos que ponemos en marcha al leer, no tendríamos en cuenta asuntos muy relevantes para esta práctica escolar, como son las intenciones y los valores que cada realidad, cada cultura ha ido demandando, tampoco se tendrían en cuenta las necesidades de cada aprendiz, siendo esto tan importante para el profesor como conocer los procesos cognitivos de un lector hábil y utilizarlos como modelo para ofrecer las ayudas específicas que necesita quien aprende. Esta separación de los dos ámbitos, el del conocimiento y el de las intenciones, ha propiciado que en el caso del lenguaje escrito, cada teoría sea manifiestamente incompleta, puesto que en cada una de ellas se han hecho preguntas diferentes y parciales.

Parece necesario asumir que las acciones educativas en tanto que acciones humanas se caracterizan por su carácter intencional y esto tanto en su dimensión individual como social. Tal carácter implica que, si toda acción humana está guiada por unas intenciones, éstas deben tener un determinado sentido, significado y valor. Admitir esto como un hecho insoslayable nos permite reconocer algunas dificultades notables para plantear teorías generales: por ejemplo la variedad de 
intenciones, que se evidencian en el hecho educativo, que son consecuencia de sistemas de valores variados y a veces contrapuestos entre quienes educan y son educados, entre quienes toman decisiones y aquellos para quienes van destinadas; por no hablar de la indeterminación de las metas para cambiar las acciones y el carácter complejo y difuso de los objetivos.

Por tanto, parece evidente que las características propias de toda acción educativa, en tanto acción humana, no permiten un análisis de ésta en términos de dicotomía teoría/práctica, tal como se utiliza en las ciencias naturales, sino que exige un tercer concepto que es el de las intenciones educativas, con toda la complejidad que ello implica. Estas condiciones suponen una relación triangular:

Figura 1. DimENSIONES DE LA ACCIÓN EDUCATIVA

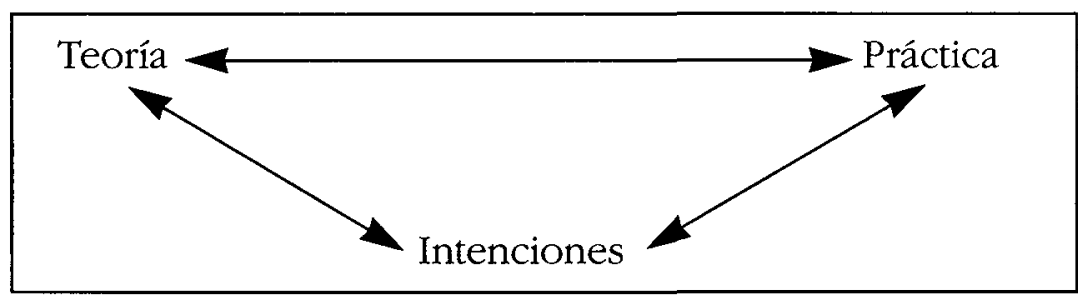

Este planteamiento equivale a admitir que toda acción educativa está basada en un componente teórico, un componente práctico y un componente dinámico (motivos, intenciones), que con demasiada frecuencia se ha ignorado o desestimado, puesto que desde el punto de vista de lo que podríamos llamar cientificismo, se ha propugnado de forma extremada la separación entre el mundo del pensamiento de los sujetos y el mundo de sus intenciones y valores. Como señala Gimeno,

El conocimiento y todo lo que puebla de significado el término teoría no puede reducirse al conocimiento científico, como ha pretendido el cientificismo positivista. Pensar, conocer, tener creencias, dar razones, reflexionar, son procesos que dan lugar a contenidos que tienen que ver con acepciones menos exigentes de la teoría $(1998,71)$.

Estas características no son exclusivas de la educación, también de muchas áreas de estudio que nos son próximas. Por ejemplo, los estudios de psicología son bastante paradigmáticos al respecto, como denuncia el propio Bruner (1991), quien, partiendo de la psicología cognitiva, ha sido capaz de reconocer y superar esa visión otorgando tanto a los valores como a la cultura un papel esencial en la explicación de la acción humana. De hecho, cuando la práctica educativa se ha guiado de forma bastante exclusivista por la psicología, primero conductista, después cognitiva, hemos visto sistemáticamente la desconsideración de las intenciones y de los valores, lo que ha llevado, aunque no fuera éste el único motivo, a producir restricciones importantes para comprender los fenómenos que se dan en la práctica educativa, inmersos como veremos en culturas con sus respectivos valores. En ciencias sociales es difícil desligar la teoría de las prácticas sociales. 
Por lo demás, en el análisis sobre la relación teoría-práctica es preciso considerar el otro aspecto relevante que enuncié al comienzo de la exposición: si la práctica es o no generadora de conocimiento, punto de vista sobre el que existen opiniones muy diversas (deductivistas e inductivistas). Para algunos teóricos de la educación, como es el caso de Carr (1996), el proceso de generación teórico surge siempre de la práctica. El autor sostiene que en educación no puede entenderse la teoría como un producto concreto de la investigación, presentada en forma de leyes y principios generales, sino sobre todo como un marco explicativo de una realidad concreta. La pedagogía es una actividad práctica, dice el autor, que a diferencia de otras disciplinas, no se ocupa sólo de elaborar teorías y explicaciones. La pedagogía es una práctica teórica por tratarse de una actividad intencional desarrollada de forma consciente, que sólo puede comprenderse en relación con el marco de pensamiento en el que sus practicantes dan sentido a lo que hacen y a lo que tratan de conseguir. Por tanto, en el estudio de lo pedagógico, y aunque no siempre los profesores son conscientes de ello, es preciso evitar cualquier distanciamiento entre teoría y práctica. A ese respecto el autor, en la citada obra, argumenta

La educación no es una especie de fenómeno inerte que pueda observarse, aislarse, explicarse y teorizar sobre él. No existen fenómenos educativos aparte de las prácticas de quienes se dedican a actividades educativas; no hay problemas educativos con independencia de los que surgen a partir de esas prácticas y no existen teorías de la educación independientes de las que estructuran y guían estas prácticas (Carr, 1996, 61).

Él mismo indica que el éxito de la teoría de la educación depende por completo de la medida en que pueda estimular a los profesores para que conozcan y comprendan mejor y más eficazmente sus propios problemas y prácticas y hacer suyas las siguientes características:

- No prescindir de su carácter práctico, en el sentido de que cualquier enfoque que teorice sus problemas desligándolos de la práctica no ayudará a resolverlos.

- Plantearse problemas que deben enunciar y resolver quienes ejercen la actividad educativa en la práctica, por lo que el valor de la teoría estará en su capacidad de estimular a los profesores a reconocer sus problemas prácticos.

- De lo anterior se deriva que las experiencias prácticas concretas deben constituir el banco de pruebas donde se evalúen los resultados de la investigación. Esto significa el rechazo a los desarrollos teóricos meramente especulativos o referidos a prácticas poco realistas.

Lo que sostiene, en definitiva, es que la legitimidad de cualquier teoría sobre la enseñanza sólo provendría de la práctica educativa: "La práctica determina el valor de cualquier teoría de la educación, en vez de que la teoría determine el valor de cualquier práctica educativa" (Carr, 1996, 60). 
Creo, sin embargo, que esta afirmación, que encierra un componente reivindicativo respecto a la importancia de la práctica, motivado en gran medida por la superioridad que tradicionalmente se ha otorgado a la teoría frente a ella, es en alguna medida exagerada. De hecho, nos puede llevar a pensar que la única legitimidad posible para investigar en educación la tienen los prácticos. Algo así como si mantuviéramos que toda legitimidad para investigar sobre la salud la tienen los médicos y debiera considerarse intrusa toda investigación que provenga de otras disciplinas básicas, como la fisiología, la bioquímica, la microbiología, etc. En este sentido, Gimeno advierte:

No aceptamos la perspectiva de que los prácticos digan qué es teoría e investigación válida a partir de su práctica y de lo que consideran útil para ella... Incluso un pragmatista como Dewey, decía: la preocupación de alcanzar alguna utilidad o práctica limita siempre la indagación científica (Gimeno, 1998, 25).

En este sentido, algunos autores, poco proclives al positivismo como Hargreaves y Goodson (1996) aun asumiendo el valor de la práctica en la propia formación de los docentes, advierten que no todo el conocimiento práctico del profesor es educativamente beneficioso o socialmente relevante, denuncian, incluso, que un excesivo celo por promover el conocimiento cotidiano, práctico, puede conducir el trabajo de los profesores fuera de compromisos morales y sociales más amplios.

Además, es preciso subrayar que la investigación que se extrae de la práctica implica procesos reflexivos, representación de la acción, un cierto alejamiento de esa práctica, se trata de esquematizar en forma de principios aquellas experiencias que son dignas de transmitir, no son la acción misma.

En definitiva, la práctica educativa no es una mera aplicación técnica: no está dirigida por un único tipo de conocimiento, el científico, sino también por las intenciones y, de forma notable, por el conocimiento tácito que proviene de la propia actividad y que, fruto de la reflexión y representación, podemos transmitir de forma más o menos codificada. La relación teoría-práctica es compleja, de eso no hay duda. Analizar esos conceptos exige ligarlos a significados amplios configurados en una perspectiva cultural, que les dé sentido y permita diferenciar distintas opiniones acerca de ellos.

En el caso de la enseñanza de la lectura debemos reconocer que el conocimiento acumulado, los grandes principios han partido esencialmente de la práctica y ha sido hasta hace unas pocas décadas la auténtica guía de las actividades de los profesores y desde donde se ha generado el conocimiento acumulado sobre el particular, si bien deberíamos igualmente admitir que éste ha sido escaso, acrítico y muy restrictivo. Hoy no es así, el conocimiento sobre el tema se ha construido desde diversos frentes y disciplinas, aunque quizás no debemos olvidar que es en gran medida desde la práctica de donde surgen la mayoría de las preguntas que nos hacemos, investigadas en ámbitos escolares o universitarios. Creo que existen ejemplos notables en este sentido. Las teorías que se denominan naturalistas han 
sido gestadas en los propios contextos escolares. Incluso, el que se cuestionara desde la teoría psicolingüística el valor de la denominada teoría perceptivista, que explicaremos en el segundo apartado, y su posterior fracaso no se debió a que Vellutino (1979) detectara sus fallos en situaciones experimentales, como mostró, por otra parte, de forma esclarecedora, sino a que las prescripciones que para la práctica se derivaban del perceptivismo no mejoraban la competencia lectora en los niños con problemas de lectura. Evidentemente las preguntas que este relevante investigador se hizo y de las que derivaron sus estudios experimentales partieron del fracaso que en las prácticas reeducadoras mostraba la teoría perceptivista.

Aunque parece necesaria la reflexión y la concienciación sobre la práctica, porque en la consciencia de las acciones se puede iniciar un proceso que nos lleva a generar conocimiento, no sólo práctico sino también teórico, debemos reconocer que el profesor no actúa en cada ocasión improvisando o creando un plan de acción, sino que aprovecha su propio conocimiento esquematizado de acciones anteriores y no necesita en cada momento apoyarse explícitamente en el conocimiento acumulado, sea éste en su dimensión cognitiva, práctica o valorativa. Es de este modo como se crean estilos y patrones docentes. El profesor, además de acumular individualmente su experiencia en la acción, puede compartir esa cultura subjetiva, de tal modo que no sólo se cree una cultura intersubjetiva que comparten un tiempo y espacio determinados, sino que se genere un proceso histórico que convierta lo subjetivo en social. Justamente esta idea entronca con uno de los propósitos de este trabajo, como es la elucidación del concepto de práctica educativa como concepto más general. La práctica educativa está constituida por saberes estratégicos, conocimientos sobre esos saberes y motivaciones compartidas. Carr (1996) advierte que "practicar siempre es actuar en el marco de una tradición", justamente porque como él mismo señala, citando a Gadamer, «lo que ha sancionado la tradición tiene una gran autoridad" y porque además la tradición no es una mera condición a la que accedemos, sino que somos nosotros quienes la producimos, sometiéndola a reinterpretación y revisión a través del diálogo y la discusión. Resumimos lo expuesto en la siguiente figura.

FIGURA 2. COMPONENTES DEL CONOCIMIENTO DEL PROFESOR

QUÉ ENSEÑAR $\longrightarrow$ SABER QUÉ
PARA QUÉ ENSEÑAR $\longrightarrow$ INTENCIONES
CÓMO ENSEÑAR $\longrightarrow$ SABER CÓMO

Si la relación entre teoría-práctica en educación es compleja, ¿podemos pensar que el conocimiento pedagógico es autosuficiente e independiente de realidades sociales y culturales? Evidentemente no, por estar inmersos en una sociedad y en una cultura, el conocimiento guarda una fuerte relación con las 
prácticas sociales en que se desarrolla. El conocimiento pedagógico se nutre de componentes que provienen de otros campos prácticos y también de otros campos teóricos. El problema está en saber cuándo estas aportaciones son pertinentes para la educación y permiten configurar un conocimiento valioso al profesor.

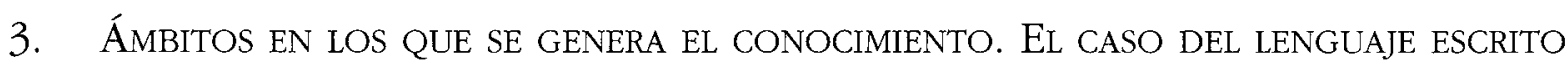

Si parece insostenible tanto una visión "teoricista" y "cientifista", que siendo externa a la práctica provea de normas a la acción educativa, como una visión "practicista", que defienda que sólo en la acción se encuentra la comprensión de lo educativo y las claves para ejercerlo, nos inclinamos por asumir que la construcción del conocimiento pedagógico ni es un campo exclusivo de los teóricos ni tampoco de las experiencias que obtiene el profesor de su práctica.

La propia complejidad de la acción educativa y su sentido dentro de una cultura o culturas nos inclina a proponer que son varios los agentes implicados en la génesis del conocimiento teórico sobre la educación y por ello que las preguntas y las respuestas pueden generarse en y desde distintos ámbitos. Una cosa es que las respuestas a problemas educativos tengan que resolverse en el ámbito escolar y otra cosa que el conocimiento que permita llevarlas a cabo provenga exclusivamente desde ese espacio concebido de forma autónoma. Sabemos de las influencias entrelazadas de diferentes saberes, de distintas procedencias, como las que existen entre las teorías de la educación y las prácticas de los profesores, pero también las que se mantienen entre la educación y prácticas sociales externas al ámbito escolar. Existen también aportaciones al conocimiento sobre educación desde otras áreas del saber, como son la psicología, la sociología, la ética, por poner ejemplos muy evidentes, sin que estas aportaciones tengan que convertirse en dependencias. Y finalmente, es innegable la influencia de los poderes políticos e incluso económicos.

La enseñanza del lenguaje escrito es un espacio ejemplar para manifestar lo que se viene planteando. En el siguiente epígrafe señalaremos algunos de estos influjos que generan conocimiento relativo a la enseñanza del lenguaje escrito, plasmados en la Figura 3. Esos influjos actúan de manera conjunta e interrelacionada en el conocimiento que el profesor utiliza cuando ejerce la práctica educativa. Los dos primeros parecen lugares más exclusivos de la génesis del conocimiento pedagógico. Los otros dos ámbitos hacen referencia a influencias de carácter social y político, pero que, sin duda, crean tendencias en el conocimiento que los profesores utilizan para enseñar a leer. 
FiguRA 3. Ámbitos QUE CONFIGURAN EL CONOCIMIENTO SOBRE LA ENSEÑANZA DE LA LENGUA ESCRITA

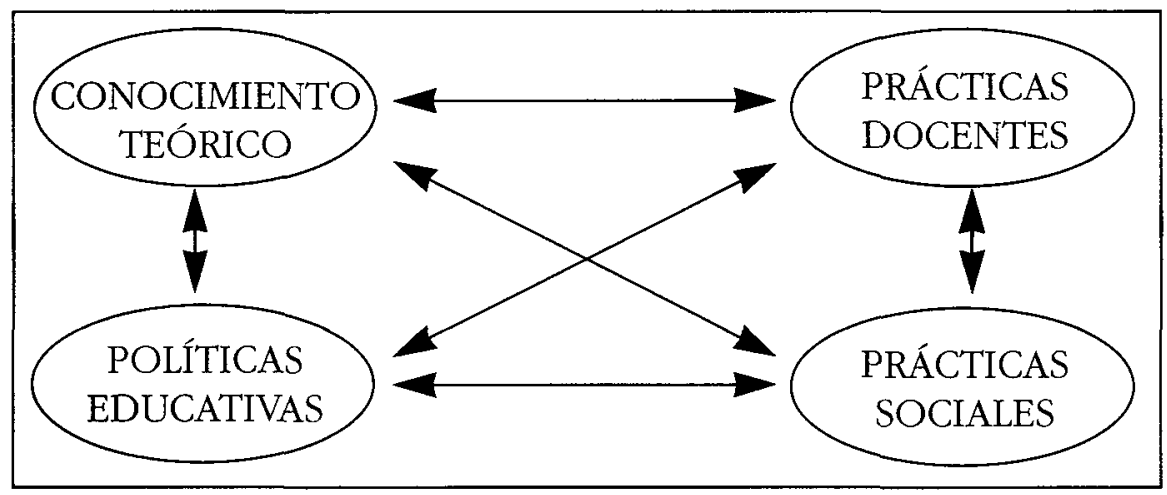

\section{a) Conocimiento teórico sobre el lenguaje escrito}

La investigación sobre el tema del lenguaje escrito, planteado desde el ámbito científico universitario, ha generado una importante aportación a los "saberes" sobre la enseñanza de la lengua escrita. ¿Cómo se ha configurado ese conocimiento teórico? ¿De dónde proceden las aportaciones? ¿Qué rasgos componen la teoría?

Ante todo, es preciso manifestar que el interesante resultado de la investigación en este campo dista mucho de configurar una teoría única y articulada. A pesar de ser un tema de estudio y reflexión con una larga trayectoria, la enseñanza de la lectura y la escritura sigue suscitando múltiples controversias en el ámbito teórico. Existen puntos de vista bastante diferentes y hasta contrapuestos acerca de qué es leer y escribir, respecto de cómo se adquieren estos conocimientos, y sobre el tipo de ayudas pedagógicas que conviene ofrecer al aprendiz. La mayor parte de los estudios sobre el lenguaje escrito se han desarrollado en la segunda mitad del siglo XX. Un notable interés por este campo de investigación viene impulsado, sin duda, cuando la alfabetización se convierte en un fenómeno extensivo (lo que nos evidencia la interrelación de los distintos ámbitos, como veremos). En ese momento se pone de manifiesto de manera inquietante que hay sujetos que no aprenden o que tienen problemas para hacerlo aun cuando están inmersos en un sistema propicio como es el sistema escolar. Incluso hay personas que no desean aprender a leer, a pesar de que se trate de un requisito básico de la escuela obligatoria. La reflexión sobre la enseñanza del lenguaje escrito sobrepasa el mundo puramente escolar y entra a formar parte de los intereses de otros "expertos", de la mirada de otras disciplinas, de manera muy especial de la psicolingüística, pero también de la sociolingüística y la neurología ente otras materias. Por ello, las teorías que configuran las explicaciones, sin duda parciales, de cómo debe enseñarse la escritura provienen de distintas visiones, de distintos campos, unas veces integrados y otras, sesgados y parcializados. No existe visión integral que explique la actividad lectora como práctica individual y social. En la base de esta parcialidad está la idea de que leer es una actividad humana con muchas implicaciones, sobre la que pueden instalarse miradas desde muchos campos. 
En un breve examen de las teorías más relevantes, lo primero que observaremos es que si cada una de ellas ha tenido respuestas singulares es porque las preguntas que han intentado responder eran distintas. Si son parciales es porque han indagado en alguno de los aspectos del tema pero no en todos. Asunto muy notable y que vuelve a entroncar con una de las premisas que se plantearon en este trabajo: las distintas realidades a las que se puede mirar cuando se plantea un problema derivan en visiones diversas.

En la segunda mitad del siglo XX el interés por el campo del lenguaje escrito sale del ambiente escolar y disciplinas como la psicología se interesan por su estudio. Las preguntas evidentemente tienen que ver con el sujeto que aprende a leer y escribir. Distintos autores se ocupan sobre todo de los que tienen problemas y empiezan a configurarse teorías al respecto. Una de esas visiones psicológicas es la llamada teoría perceptivista. Esta teoría, preocupada por los problemas para aprender a leer en determinados sujetos, sostiene que dichos problemas provienen de una iniciación prematura de esa enseñanza, cuando el niño no tiene aún la madurez necesaria en una serie de factores, que se presumen relacionados con la lectura, y que son en buena parte de carácter perceptivo (discriminación visual y auditiva, orientación espacial, etc.); y alguno de ellos también con la lengua oral, como, por ejemplo, la pronunciación de palabras, etc. (Downing y Thackray, 1974). La derivación práctica de todo ello es el entrenamiento para que los aprendices maduren en esas destrezas, lo que se hace extensivo a los lectores con problemas. Lo cual genera la proliferación de materiales al respecto y la aparición de pruebas de madurez lectora.

El fracaso de la teoría perceptivista queda patente cuando se evidencia que esas prácticas no mejoran la competencia lectora de quienes tienen problemas; además, sus fallos se hacen evidentes en los estudios experimentales de Vellutino (1987), quien muestra que los malos lectores no lo son por deficiencias en los factores señalados, sino que su problema proviene de fallos en los procesos lingüísticos que intervienen en el reconocimiento de la palabra escrita en el sistema alfabético. De ahí que el problema retorne a la psicología y se convierta en objeto de estudio de un buen número de investigadores del campo de la psicolingüística. Nace así a partir de los setenta una fuerte corriente de investigación cognitiva en lectura. Grupos de investigadores hacen del estudio del aprendizaje de la lectura su gran reto.

La teoría psicolingüística ha centrado su atención en el estudio de los procesos cognitivos involucrados en la lectura, analiza cuáles son las vías de acceso al léxico interno al leer; subraya las diferencias entre expertos y novicios, identifica los componentes que funcionan mal en aquellos individuos que muestran problemas en este aprendizaje, el papel que juega la fonología en el aprendizaje del lenguaje escrito en los sistemas alfabéticos. Todo ello bajo el supuesto de que esos procesos son idénticos en cualquier contexto, y con la convicción de que es posible analizar objetivamente al sujeto lector, sin tener que atender a su medio social 
ni a la cultura a la que pertenece. Un análisis detallado de esta teoría puede verse en Vellutino (1979); Cuetos (1990); Morais (1998).

La posición de esta línea respecto de la enseñanza es clara: conocidos los componentes de la lectura y la escritura, diseñemos programas para que el alumno se apropie y automatice esos componentes. Para algunos incluso se trataría de potenciar habilidades altamente estructuradas a partir de materiales muy específicos para llevarlas a cabo. Entre las limitaciones del modelo cabe señalar, por ejemplo, la desconsideración del contexto tanto de aprendizaje como social y desde luego el valor cultural de ser lector. En suma, esta teoría se preocupa de la enseñanza del código, pero se despreocupa de cuestiones como: ¿cuáles son las funciones que puede tener dominar el lenguaje escrito para un aprendiz de cuatro o cinco años? ¿Por qué va a interesar a un niño aprender a leer? ¿Qué tipo de lector queremos? ¿Qué alfabetización es suficiente y para qué?

Aunque esta visión, así expuesta, proviene de estudios mayoritariamente ajenos al ámbito escolar, es preciso reconocer que viene a respaldar muchas de las prácticas escolares. Una tradición que proviene de la antigua Grecia, en sus modalidades más rigurosas, donde como todos sabemos se inventó el alfabeto.

Otro grupo de teorías son las denominadas teorías comunicativas. En las que incluiremos el lenguaje integrado (Goodman, 1995) y las propuestas constructivistas. Los primeros defienden de manera más o menos drástica una apropiación natural del lenguaje escrito, a semejanza de como se desarrolla el lenguaje oral, incluso integrado con éste. Las premisas fundamentales de esta visión podrían ser éstas: se aprende a leer y escribir como se aprende a entender y hablar de forma oral; aprender supone que el individuo realice interacciones con un medio letrado, con un entorno de materiales impresos; el niño construye su aprendizaje de forma activa en un proceso de interacción con textos y según se suceden los estadios de su propia madurez para ese aprendizaje. Por tanto, se propiciará la espontaneidad del aprendiz y se reducirá al mínimo el papel del docente que debe limitarse a facilitar materiales, textos, que el niño debe explorar y, a partir de esa interacción individual con los textos y de su propio desarrollo, el pequeño construirá ese conocimiento. La mayor ventaja de este enfoque estaría, según sus defensores, en el valor que otorga al que aprende como sujeto activo, así como el sentido funcionalista que tendría para él este aprendizaje, lo que propiciaría una mayor significatividad. Estas teorías, como se puede intuir, cargan las tintas en el aspecto intencional de la práctica, importa sobre todo el para qué, pero curiosamente se plantean de forma débil el qué, y de forma un tanto indefinida el cómo.

Sus desventajas, fallos o lagunas, provienen sobre todo de la identificación que realizan entre lenguaje oral y escrito y por tanto en el modo de aprender uno y otro; el énfasis individualista que dan al aprendiz; la idea subyacente de que estos estadios son también universales (como eran los procesos en las anteriores teorías), desconsiderando las prácticas diferentes de las distintas culturas. Con todo, lo más sorprendente es la falta de explicación acerca de cómo se aprende a leer, así como 
del papel que tiene el docente como mediador del aprendizaje; papel que reducen tan sólo a facilitar un contexto de materiales escritos.

Dentro de planteamientos funcionalistas, el constructivismo, en una línea semejante en ciertos aspectos, defiende que los niños construyen la idea de qué es la escritura a través de hipótesis y comprobaciones según sus experiencias con materiales escritos. Esta teoría se configura a partir del constructivismo, como teoría del aprendizaje y sus hipótesis son contrastadas no en situaciones experimentales, sino escolares (Tolchinski, 1993; Teberosky y Tolchinski, 2004).

Ambas tendencias desaprueban cualquier tarea instructiva que tenga que ver con la enseñanza del código, por considerar que esto es innecesario, que el pequeño lo descubrirá en su experiencia con la propia escritura, sin admitir que éste es precisamente el gran escollo en la mayor parte de los casos que presentan problemas serios para aprender a leer.

La teoría sociocultural, por su parte, plantea que el lenguaje escrito es un invento social, de carácter cultural y por ello su aprendizaje tendrá lugar a partir de un proceso de interacción y ayuda de los miembros formados de una cultura a los que no lo estén. Este paso, que se denomina andamiaje, supone un auténtico proceso de mediación entre los docentes y los alumnos, hasta que éstos dominan ese conocimiento y pueden actuar de forma autónoma. Vigostky (1979) desarrolló una compacta teoría sobre el lenguaje escrito y su papel en el desarrollo de los procesos cognitivos, aunque pudo desarrollar sólo de forma esquemática algunas indicaciones sobre cómo enfocar su enseñanza, planteando una transición por distintos sistemas de representación más elementales de los que dispone el ser humano hasta llegar a la escritura.

Las consideraciones pedagógicas que extraemos de este último planteamiento son que el aprendizaje del lenguaje escrito está totalmente relacionado con la cultura en que esté inmerso el sujeto que aprende, es decir, con los usos y funciones que para su contexto tiene este conocimiento. En esta corriente el diálogo juega un papel clave y no se le considera como algo introductorio y facilitador, sino como el eje del proceso de construcción del conocimiento, en este caso, de la alfabetización. Profesores y alumnos son igualmente importantes y, sobre todo, igualmente activos en el proceso. Tanto de textos de Vygotski $(1979,1982)$ como de Bruner $(1984,1991)$ extraemos planes o ideas pedagógicas concretas que van más allá de la idea genérica e imprecisa de sumergir al pequeño en un medio letrado, como defienden las teorías naturalistas.

Las propuestas integradoras han sido planteadas por autores como McCarthey y Raphael (1994); Pressley (1999), entre otros, señalando que las tres teorías, que podemos llamar respectivamente procesamiento de la información, naturalista y socioconstructivista, pueden operar conjuntamente para construir una imagen de los procesos convergentes de la lectura y la escritura. En dicho planteamiento se trabajarían tanto los aspectos funcionales como las habilidades lingüísticas. 


\section{b) Las prácticas docentes}

La enseñanza de la lectura ha sido una de las prácticas educativas, y sociales, más relevantes en la historia de la educación en el siglo XX (Viñao, 1999) por lo que el conocimiento teórico sobre el lenguaje escrito no puede ser ajeno a la influencia que tales prácticas han tenido a lo largo del proceso histórico de enseñanza de la lectura y la escritura, y consecuentemente a la acción de los profesores, por lo cual la práctica es otra de las fuentes esenciales, igualmente cruzada, como señalé en la Figura 3.

Desde ese interés práctico las preguntas esenciales que se han formulado los profesores tienen que ver, ante todo, con la idea de cómo ayudar al niño a apropiarse del sistema de escritura, cómo motivarlo para aprender el lenguaje escrito, qué materiales facilitarían tal propósito.

Como señalé en relación con el terreno de los teóricos, también en el de los prácticos ha habido y hay notables discrepancias. Hay que reconocer que este asunto ha sido siempre un objeto de especial interés para el profesorado en el último siglo, no hay más que ver las "modas" que han existido y coexistido sobre la forma más acertada de enseñar el lenguaje escrito. Las tradiciones sobre los llamados métodos analíticos y sintéticos, su interés o eficacia, su adecuación o no a los intereses infantiles, han sido planteamientos que han repercutido en el conocimiento acumulado sobre la lectura y la escritura. La persistente tradición fonetista en la enseñanza del lenguaje escrito desde la aparición del sistema alfabético, planteada, eso sí, de forma más o menos afortunada, pone de manifiesto un tipo de prácticas que se consideran necesarias como son las de enseñar el código, aunque algunas tendencias nieguen este supuesto. Podríamos nombrar innumerables programas nacidos de la práctica, métodos, materiales, surgidos de las intuiciones de maestros enfrentados a problemáticas particulares, que han legado sus propuestas, a veces seguidas ortodoxamente, otras transformadas acertadamente por otros maestros.

En la búsqueda para facilitar al niño la relación entre fonemas y letras, se han realizado propuestas con apoyos distintos. Desde los métodos cromáticos, Gattegno (1969) y Gray (1956) a los gestuales como el método Sanabria (Martín Sanabria, 1952), sin olvidar materiales tan vistosos como, por ejemplo, el Abecedario Iconográfico en formato cartel de Saturnino Calleja Fernández, donde se asocia cada letra con un objeto, cuya posición "recuerda" a la letra representada, todos ellos intentando facilitar el acceso a un sistema claramente artificial, intentando motivar para un conocimiento que ha separado demasiadas veces los aspectos formales de un instrumento esencial, de los funcionales. Conocemos propuestas del año 1935 contrarias a la visión fonetista, defensores de procedimientos globales, semejantes a los planteados por Decroly como la Metodología de la lectura y la escritura de Doreste (1935).

Insignes pedagogos, reconocidos también por sus visiones sobre otros muchos aspectos educativos, han realizado propuestas para enseñar a leer, tratando de llevar al aprendiz cualquiera que fuera su edad por caminos fáciles y motivadores. 
Los planteamientos de Freinet sobre la lectura han tenido una influencia sustancial en el ejercicio práctico de cientos de profesores y aunque ahora pudiéramos darles justificaciones teóricas o incluso hacer críticas a algunas de sus técnicas, precisamente desde el conocimiento teórico, lo claro es que surgieron desde y para la práctica respondiendo a las preguntas igualmente prácticas arriba recordadas y que han permitido a cientos de niños no sólo en su país de origen (Francia) aprender a leer con gusto e interés.

El método de alfabetización de Freire surge igualmente buscando formas de motivar e introducir a los adultos analfabetos en el lenguaje escrito. Se plantea, pues, desde la práctica y claramente imbricado en una perspectiva tan social como educativa. Grupos de profesores han generado, en nuestro país, materiales que surgidos desde su experiencia práctica han sobrepasado con creces su contexto para ser utilizados por miles de profesores, a veces como fuente única. Incluso superando la visión inicial de apropiación del código alfabético, también desde agentes cercanos a la práctica han surgido propuestas sobre comprensión lectora, acertadamente coincidentes con las interesantes aportaciones teóricas, tratando de aunar en el tiempo ambos procesos. Contamos con los acertados planteamientos de Cairney (1992) y Wray y Lewis (2000).

A pesar de considerar las prácticas de los profesores como un elemento configurador del conocimiento, tal influjo se realiza de forma un tanto asistemática, debemos reconocer que los estudios sobre las prácticas de aula en relación con la enseñanza de la lectura son más bien escasos, así se recoge por Langer y Allington (1992) en una amplia revisión sobre investigación y prácticas escolares relacionadas con la lengua escrita. En este trabajo se viene a reconocer que la investigación en lectura se ha llevado a cabo sobre todo desde el ámbito teórico y especialmente desde la perspectiva psicolingüística.

\section{c) Politicas educativas}

Aunque en principio muchos cuestionarían que este asunto pueda relacionarse con el conocimiento teórico, lo cierto es que las prescripciones que frecuentemente difunden los documentos inspirados en unas $u$ otras políticas educativas tienen, sin duda, una fuerte influencia en las ideas, imágenes, principios y reglas prácticas que configuran lo que podríamos denominar el conocimiento docente, al menos en lo que respecta a sus teorías implícitas. De hecho las decisiones que provienen de las políticas educativas son una potente influencia que conduce a generar usos en la práctica que no provienen ni del conocimiento teórico ni del práctico, aunque en ocasiones se busque cobertura o cierta legitimidad sobre todo en el teórico. Tenemos ejemplos prácticamente de todos los colores y épocas, aunque me limitaré a reseñar dos de las últimas décadas y leyes.

Uno de ellos lo tenemos en una idea surgida en el transcurso del desarrollo de la LGE de 1970: la propuesta de no enseñar a leer antes de los seis años, que 
contravenía la vieja tradición de enseñar a leer tan pronto se escolarizaba a los niños, por ejemplo, ya en el parvulario llamado, después, escuela infantil. Quizás conviene recordar que a partir de la década de los cincuenta era bastante normal que muchos niños empezaran a escolarizarse a los cuatro años en las llamadas Escuelas de Párvulos ( 4 a 6 años) y era en esta etapa cuando se iniciaba como algo habitual la enseñanza del lenguaje escrito, pues se pretendía que los escolares salieran normalmente leyendo cuando concluían dicha etapa, que no era obligatoria. No perdamos de vista que la alfabetización se apreciaba como la llave de todo aprendizaje escolar y por ello se hacía necesario saber leer y escribir para proseguir la enseñanza obligatoria después de los seis años. Pues bien, a través de la LGE (Ley General de Educación de 1970), se da la consigna de que tal práctica debe cambiar en el sentido de retrasar esta enseñanza hasta los seis años, lo que coincide con el momento en que la escolarización se hace obligatoria. La justificación esencial de esta prescripción desde la política educativa proviene probablemente de una razón social clara: si la enseñanza de la lengua escrita es básica para la escolaridad posterior, parece justo que tal enseñanza tenga lugar en una etapa obligatoria, puesto que tal cosa se ajusta a una política de igualdad de oportunidades.

La razón puede ser en sí misma poderosa, sin embargo, se la reviste de una justificación psicopedagógica, que brinda la teoría perceptivista, que describimos antes, y que viene a refrendarse con argumentos "científicos" tales como que el niño no está "maduro" para aprender a leer antes de esa edad, que antes de iniciarse la enseñanza debe ser preparado en una serie de aspectos de orden diverso (percepción, orientación espacio-temporal, desarrollo del lenguaje oral, etc.). Todo ello dio lugar a que la etapa de párvulos, ya denominada educación preescolar, se convirtiera en este orden de cosas en un tiempo de ejercitación en ese tipo de destrezas, que supuestamente prepararían al pequeño aprendiz para que a los seis años estuviera "maduro" para la aventura de aprender a leer. Curiosamente buena parte de los argumentos de la teoría perceptivista fueron desmentidos después por otras teorías y planteamientos, como señalamos antes: la teoría psicolingüística desdijo con pruebas de enorme relevancia que la lectura y los problemas que algunos sujetos tienen para adquirirla se debieran a causas perceptivas. Además, retrasar la edad de inicio fue cuestionado e incluso censurado por planteamientos tan distantes, entre sí, como los que mantenían ya en los ochenta Cohen (1983) y Doman (1978) a favor del aprendizaje temprano de la enseñanza de la lectura y los manifestados por Vigotsky (1979), quien subrayó esa misma opinión con otro tipo de razones. En todo caso es preciso resaltar que las prescripciones lanzadas desde la Administración Educativa calaron hondamente en el pensamiento de muchos profesores, e incluso se consideró poco progresista enseñar a leer al niño en preescolar.

Otro ejemplo notable, supongo que muy presente aún, dada la cercanía temporal del mismo, es la prescripción que se hace en el Proyecto Curricular Base derivado de la LOGSE sobre cómo debe ser la enseñanza de la lectura. En este caso la prescripción viene de la mano de planteamientos constructivistas, dado que seguramente los asesores en la materia tenían esta tendencia. En todo caso lo que 
interesa resaltar es el hecho de que se formule con gran precisión cómo debe enfocarse esta materia, incluso el tipo de actividades a realizar. Evidentemente los poderes políticos influyen, sin duda, más allá de lo razonable en las prácticas del aula, conectados con agentes educativos que diseminan sus prescripciones, lo que, por otra parte, otorga legitimidad pedagógica.

\section{d) Prácticas sociales}

Imbricados con la presión de las políticas educativas, los grupos sociales son importantes agentes de influencia. En el lenguaje escrito los ejemplos son elocuentes. La idea de alfabetización ha variado a lo largo de la historia tanto en su sentido como en su extensión, planteando exigencias escolares diferentes en esa evolución. No es preciso remontarse a quienes ya enseñaban a leer en Mesopotamia, Grecia o en la Edad Media, cuyas culturas y estructuras sociales determinaban a quién se enseñaba, cuáles eran los métodos y qué textos se seleccionaban para la enseñanza (Cavallo y Chartier, 1998).

Ejemplos de la actualidad y del pasado inmediato pueden ser igual de ilustrativos. Lo que se entendía en España en los años cuarenta y cincuenta como un lector medio hoy sería un lector insuficiente. Recordemos que, en esas décadas, estamos hablando de una sociedad con índices de analfabetismo del 41,4\% en el caso de los hombres y del 50,6\%, en el de las mujeres en 1920 y de $17,2 \%$ en los hombres y el $23,1 \%$ en las mujeres todavía en 1940 , por lo que es evidente que el objetivo de una alfabetización en ese momento consistía en que los aprendices conocieran el sistema alfabético y fueran capaces de poder leer, a veces simplemente descifrar, textos muy elementales, útiles para funciones vitales básicas. De ahí que los planes para hacer lectores fueran denominados planes de alfabetización en su sentido más estricto, como fue la puesta en marcha del plan del año 1922 y el de 1950, destinados a una sociedad tradicional que iba paulatinamente emigrando del campo a la ciudad y cuyo objetivo era tan básico como enseñar a leer a los muchos analfabetos entonces existentes, pretendiendo con ello poco más que ayudarles a descifrar textos (Viñao, 1999).

Cuando en años posteriores se consideró que estos niveles de alfabetización era altos, se cambió incluso la denominación de tales programas y se sustituyeron por otros más amplios y algo más ambiciosos, como fueron los programas de educación y promoción de adultos, de 1963 y 1973, sucesivamente, cuyo título era Campaña Nacional de Alfabetización y Promoción Cultural de Adultos, estaban destinados a una población que debía ocupar los nuevos puestos en la incipiente industria que se desarrollaba en el país. Los planes para que la lectura no fuera un mero y primario instrumento práctico sino un ejercicio placentero, un instrumento de formación, estaban todavía un poco lejos.

Ahora también ha variado esa idea de lector. El ascenso social se vincula hoy con otros instrumentos en gran medida relacionados con la enseñanza del lenguaje 
escrito, como por ejemplo, la informática; de hecho se habla de alfabetización informática, como una necesidad perentoria. Hoy necesitamos lectores competentes que usen el texto escrito para realizar funciones nuevas, ampliando y mejorando las anteriores. Leer para aprender requiere niveles de competencia mucho más altos que los exigidos hasta no hace mucho. El uso de la lectura en el tiempo libre genera que uno de los aspectos en que tiene que insistir la escuela es en crear el gusto por leer y la competencia para hacer lectores mejores. La nueva dimensión en la que nos introducen las nuevas tecnologías hace necesario formar expertos no sólo en leer textos sino en seleccionarlos, componer nuevas formas de comunicación a través de mensajes escritos (chat, correo electrónico, etc.). Es interesante al respecto el análisis que hace Cassany (2006) sobre lo que denomina "lectura contemporánea". La alfabetización hoy es otra cosa y la escuela debe dar respuesta a esa nueva concepción.

Podemos ver, pues, que otro tipo de conocimiento que utiliza el profesor viene en gran medida condicionado por la cultura en la que la práctica lectora tiene lugar, lo que implica una forma de entender la alfabetización.

\section{COMPONENTES DE UNA TEORÍA DE LA ENSEÑANZA DEL LENGUAJE ESCRITO}

Resumiendo y admitiendo, al menos, las influencias descritas, es preciso reconocer que el tipo de saber teórico que necesita el profesor y el papel que tiene en su propia práctica es, pues, un saber hacer compuesto de formas de saber cómo, aunque ligado a creencias, a motivos y a valores. Esa tradición se transmite en forma de cultura pedagógica en su triple dimensión: descriptiva o teórica, el saber qué, constituido por las representaciones tanto ficticias como científicas; el saber cómo, que se concreta en técnicas, instrucciones y métodos y el saber para qué, los valores e intenciones (Figura 4). Todo ello da lugar en realidad a lo que llamamos teoría educativa. Éste es el modo, sin duda, de dar continuidad y asegurar la comunicación entre las acciones subjetivas, cargadas de valor experiencial y el mundo de lo social.

FIGURA 4. COMPONENTES DEL CONOCIMIENTO PARA LA ENSEÑANZA DE LA LENGUA ESCRITA

$\begin{array}{ll}\text { QUÉ ENSEÑAR } & \text { ALFABETIZACIÓN } \\ \text { PARA QUÉ ENSEÑAR } \longrightarrow \text { FUNCIONES } \\ \text { CÓMO ENSEÑAR } \longrightarrow \text { TAREAS DIDÁCTICAS }\end{array}$

De acuerdo con todo lo expuesto y debatido, es preciso asumir que en relación con una teoría para la enseñanza de la lectura, como ejemplificación de teoría de la enseñanza, deberemos tener en cuenta varios aspectos: 
1) Estará configurada por distintas influencias y saberes, dando cabida a conocimientos externos a la misma.

2) Estará constituida por visiones o teorías parciales, no necesariamente articuladas.

3) Tendrá presentes los aspectos dinámicos, las intenciones que están en la base del hecho educativo.

La primera conclusión supone admitir que el conocimiento teórico sobre la enseñanza del lenguaje escrito se configura a partir de fuentes distintas: las que provienen de disciplinas externas a la educación, y la investigación que en ellas se genera, algo que se defiende por quienes sostienen que la teoría de la educación se nutre de fuentes externas. El conocimiento generado en la práctica, que se transmite de manera bastante inconsciente, pero que constituye una tradición ineludible para comprender por qué los profesores realizan determinadas prácticas. Factores sociales y políticos que ejercen influencias de manera muy sutil y que sin ser propiamente conocimiento teórico llegan a influir decisivamente en el conocimiento implícito de los profesores.

La segunda cuestión, como hemos visto, nos permite confirmar que, como sostiene Cartwright (1999), las teorías científicas distan mucho de ser globales. La búsqueda de teorías generales y de una visión científica unívoca es probablemente más utópica, si no inalcanzable.

Las teorías de la educación cuentan con un componente que va más allá del carácter explicativo que busca cualquier ciencia, es su carácter intencional, teleológico y axiológico. Educamos con intenciones y éstas se basan en valores determinados.

Son pues algunos rasgos del conocimiento teórico de la enseñanza del lenguaje escrito que probablemente pudieran extenderse a otras teorías de la enseñanza.

\section{BIBLIOGRAFÍA}

Bruner, J. (1984) Acción, pensamiento y lenguaje. Madrid, Alianza.

- (1986) (2004) Realidad mental y mundos posibles. Barcelona, Gedisa.

- (1991) Actos de significado. Más allá de la revolución cognitiva. Madrid, Alianza Psicología Minor.

CAIRNEY, T. H. (1992) Enseñanza de la comprensión lectora. Madrid, Morata.

CARR, W. (1996) Una teoría para la educación. Hacia una investigación educativa crítica. Madrid, Morata.

Carrasco, J. y García del Dujo, A. (2002) La teoría de la educación en la encrucijada, Teoría de la Educación. Revista Interuniversitaria, 13, 15-43.

Cartwright, N. (1999) The Dappled World. Cambridge University Press.

CASSANY, D. (2006) Tras las líneas. Sobre la lectura contemporánea. Barcelona, Anagrama.

CAVAllo, G. y Chartier, R. (1998) Historia de la lectura en el mundo occidental. Madrid, Taurus. CoHen, R. (1983) En defensa del aprendizaje precoz. Madrid, Cincel-Kapelusz. 
COlom, A. y RinCón, J. (2004) Epistemología neoidealista y fracaso fundacional del saber educativo, Teoría de la Educación. Revista Interuniversitaria, 16, 19-48.

Cuetos, F. (1990) Psicología de la lectura. Madrid, Escuela Española.

Doman, G. (1978) Cómo enseñar a leer a su bebé. Madrid, Aguilar.

Doreste, F. (1935) Metodología de la lectura y escritura. Madrid, Publicaciones de la Revista de Pedagogía.

Downing, J. y Thackray, D. V. (1974) Madurez para la lectura. Buenos Aires, Kapelusz.

GatTeGno, C. (1956) Words in Colour. G. B. Training Film, Educational Explorers.

Gimeno, J. (1998) Poderes inestables en educación. Madrid, Morata.

GoOdman, K. S. (1995) El lenguaje integral: un camino fácil para el desarrollo del lenguaje, Textos, 3, 77-91.

GRAY, W. S. (1956) La enseñanza de la lectura y la escritura. Ginebra, UNESCO.

HACKING, I. (1996) Representar e intervenir. México, Paidós.

Hargreaves, A. y Goodson, I. (1996) Teacher's Professional Lives: aspirations and actualities, en Goodson, I. y Hargreaves, A. (eds.). Teachers Professional Lives. London, Falmer Press, 1-27.

LANGER, L. y AlLINGTON, R. (1992) Curriculum research in writing and reading, en JACKSON, Ph. W. (ed.). Handbook research of curriculum. New York, MacMillan Publishing Co, 687725.

Martín Sanabria, M. (1974) Sistema onomatopéyico. Madrid, Gráficas Aguirre Campano.

McCarTheY, S. y RAPHAel, T. (1994) Perspectivas de la investigación alternativa, en IRWIN, J. y DOYLE, M. Conexiones entre lectura y escritura. Montevideo, Aiqué.

MORAIS, J. (1998) El arte de leer. Madrid, Aprendizaje-Visor.

Pressley, M. (1999) Cómo enseñar a leer. Barcelona, Paidós.

SARRAMONA, J. (2003) La perspectiva tecnológica en la acción educativa, en VV.AA. Teoría de la Educación ayer y boy. Murcia, 159-204.

TEBerosky, A. y TOLCHINSKI, L. (2004) Más allá de la alfabetización: el conocimiento fonológico, la ortografía, la composición de textos, la notación matemática y el aprendizaje. Buenos Aires, Santillana.

Tolchinski, L. (1993) Aprendizaje del lenguaje escrito, procesos evolutivos e implicaciones didácticas. Barcelona, Anthropos.

Vellutino, F. R. (1979) Dyslexia. Theory and Research. Cambridge Mass, MIT.

- (1987) Dislexia, Investigación y Ciencia, 128, 12-20.

VIGOTSKY, L. S. (1979) El desarrollo de los procesos básicos superiores. Barcelona, Grijalbo.

VIÑaO, A. (1999) Leer y escribir. Historia de dos prácticas culturales. Madrid, Fundación Educación.

WRAY, D. y LEWIS, M. (2000) Aprender a leer y escribir textos de información. Madrid, Morata. 